\title{
Virtopsia. Aplicaciones de un nuevo método de inspección corporal no invasiva en ciencias forenses.
}

\author{
Virtopsy. Applications of a new method of non-invasive \\ body inspection in forensic sciences.
}

J. Aso', JV. Martínez-Quiñones'1, J. Aso-Vizán'4, J. Pons², R. Arreguili y

S. Baena ${ }^{3}$

\section{RESUMEN}

Los modernos métodos de imagen, como la CT y la RNM proporcionan datos reales y tridimensionales de la superficie y las estructuras internas corporales. Esta información se almacena en ficheros DICOM (un protocolo estándar en adquisición de imagen radiológica). Sobre esos ficheros pueden realizarse muchos procedimientos de análisis de imagen, tales como visualización interactiva, morfometría, reconstrucciones tridimensionales, etc. Estas tareas son realizadas de una manera no invasiva y no destructiva, pudiéndose almacenar los resultados para documentación del caso. El presente trabajo describe las bases de los procedimientos de análisis de imagen sobre ficheros DICOM dirigidos a propósitos forenses (la llamada "virtopsia"), enfatizando la utilidad del método en Patología, Traumatología Forense y otros campos.

La "virtopsia" debe ser considerada no sólo como un procedimientos post-mortem (complementando pero no sustituyendo a la autopsia tradicional), sino como un modo de examinar partes corporales de una manera interactiva, sea en el fallecido o en el sujeto vivo. Ello amplia su utilidad y hace apropiado el procedimiento siempre que se requiera un examen anatómico forense reproducible, preciso, interactivo y en tiempo real.

Palabras clave: Análisis de imagen, Proceso de imagen, ficheros DICOM, Radiología digital, Autopsia, Virtopsia, Radiología cadavérica, Radiología asistida por ordenador.

\section{ABSTRACT}

Modern imaging methods, such as radiological CT/MRI scanning provides real, full 3D data of the body surface and internal structures. This information is stored in DICOM files (a standard protocol in radiology image acquisition). Many image-analysis procedures, such as interactive visualization, morphometry, densitometry, three dimensional reconstruction, etc., can be carried-out on these files. These tasks are performed in a non-invasive and non-destructive manner, and the results can be stored in order to document the case.

The present paper describes the basis of imageanalysis procedures on DICOM files directed to forensic purposes (socalled "virtopsy"), emphasizing the utility of the method in Pathology, Forensic Traumatology and other fields.

The virtopsy must be considered not only as a postmortem procedure (complementing but not substituting the traditional autopsy), but a way to examine body parts in an interactive manner, whatever the subject is dead or alive. That spreads its utility and makes the method appropriate whenever an accurate, real-time, interactive and reproducible forensic anatomic examination is required.

Key words: Image analysis, Image processing, DICOM files, Radiology, Autopsy, Virtopsy, Post-mortem Radiology, Computerassisted Radiology.

Cuad Med Forense 2005; 11(40):95-106

Fecha de recepción: 23.ENE.06

Fecha de aceptación: 26.ENE.06

Correspondencia: Servicio de Neurocirugía. Hospital MAZ. Avenida de la Academia General Militar, 74. 50015-Zaragoza. E-mail: jaso@maz.es

1 Servicio de Neurocirugía. Hospital MAZ. Zaragoza.

2 Servicio de Radiología. Hospital MAZ. Zaragoza.

3 Instituto de Medicina Legal de Aragón.

${ }^{4}$ Facultad de Medicina. Universidad de Zaragoza. 


\section{INTRODUCCIÓN:}

Durante los últimos años, uno de los mayores avances en Medicina ha venido protagonizado por el auge de las llamadas técnicas de imagen, principalmente la TC y la RNM.

Pese a su denominación, estos procedimientos no obtienen sólo imágenes reales del interior del cuerpo, sino ficheros informáticos que contienen, aparte de los datos que permiten reconstruir una región anatómica, información sobre muchos otros aspectos como densidades, calibraciones, condiciones de la captura, etc.

Esta información se almacena en un protocolo estándar que son los llamados ficheros DICOM.

Sobre dichos ficheros pueden realizarse todo tipo de tareas de análisis y proceso de imagen (API), permitiendo examinar una parte corporal de una manera muy precisa e interactiva, aplicando sobre ella técnicas reconstructivas o mensurativas sin necesidad de alterar el modelo del que proceden.

Desde hace un tiempo, y fomentado en parte por el declinar del número de autopsias en medios hospitalarios, se ha preconizado el empleo de procedimientos alternativos a la autopsia tradicional que han dado en llamarse "opsias" [I]. Ejemplos son las ecografías post-mortem (ecopsias), la endoscopia cadavérica (endopsia), y otros que utilizan, por ejemplo, técnicas radiológicas (radioscopsias).

Recientemente, se ha acuñado el término "virtopsia" [2,3] para denominar el conjunto de procedimientos de API con finalidad forense, que pueden realizarse sobre ficheros obtenidos mediante radiología digital. Tales técnicas tienen aplicaciones en Patología y también en Clínica Forenses.

La ventaja es que podemos estudiar el cuerpo humano o una región anatómica en tiempo real, sin abrir o mutilar el cuerpo y hacerlo, además, interactivamente. La virtopsia puede recaer sobre un cadáver o una persona viva, y permite, entre otras cosas, detectar detalles ocultos, conocer las propiedades de los tejidos, investigar las modalidades de lesión en modelos tridimensionales sin alterarlos, y un largo etcétera de aplicaciones forenses.

Basándonos en la experiencia que nos da el empleo rutinario de estas técnicas en el sujeto vivo [4] y su utilización en algunos casos de fallecidos, el presente trabajo pretende enfatizar la enorme utilidad de estas técnicas y sus aplicaciones como complemento de la autopsia tradicional y también en el ámbito de la Clínica forense.

\section{TÉCNICAS DE VIRTOPSIA:}

Como hemos señalado, la virtopsia no es sino el resultado, en el cadáver o en el vivo, de procedimientos de API con una finalidad forense.

Las técnicas de API pueden definirse como el conjunto de procedimientos de índole manipulativo o mensurativo que se aplican sobre una imagen digital con una finalidad científica.

Estos procedimientos han sido utilizados en Tanatología, Patología y Traumatología Forenses $[5,6,7,8,9,10,1$ I ] . En Histopatología, su utilización se ha extendido desde la morfometría de diferentes estructuras [ 12 ] hasta sofisticadas aplicaciones en citogenética [13]. En criminalística algunas de estas técnicas han sido empleadas para el revelado de huellas y marcas de zapatos [14]. Entre las aplicaciones en Antropología forense destacan la estimación de la edad de restos óseos a través de los dientes [15, I6], o los estudios comparativos en cráneo a efectos identificativos [17]. 
Igualmente, su utilidad en la valoración lesional dentro del amplio campo de la valoración de daño corporal (VDC) es enorme, como tendremos ocasión de enfatizar más adelante.

La virtopsia usa las técnicas de API sobre imagen digital procedente de dispositivos radiológicos de imagen (principalmente TC y RNM). Analizaremos ahora los ficheros en que esta imagen queda almacenada, para pasar después a ver las principales técnicas.

\section{Ficheros DICOM:}

Un fichero DICOM puede verse como un lote de imágenes que son descritas y ordenadas por un fichero de índice, denominado DICOMDIR. Los segmentos que componen la pila son cada uno de los cortes que el TC o la RNM han producido. Usualmente, hay tantas listas (pilas) como áreas de cortes paralelos entre sí. Ello posibilita que, mediante software apropiado, puedan reconstruirse regiones corporales en tres dimensiones.

Cada fichero DICOM consta de una cabecera, en la que figuran datos del paciente junto con otros de la adquisición (tipo de técnica de imagen, calibración, etc.), y de datos de imagen (información en dos dimensiones o en tres dimensiones) [18].

En realidad el acrónimo DICOM no se refiere a una definición de un fichero gráfico. El término contiene las iniciales de: "Digital Imaging and Communications in Medicine", y especifica una serie de protocolos y formatos gráficos para el intercambio de imágenes, datos e información asociada para aplicaciones médicas.

El protocolo DICOM contiene, pues, diferentes elementos que, en una gran mayoría, son imágenes con información asociada, ambos encapsulados en ficheros susceptibles de ser sometidos a intercambio y lectura por estaciones DICOM (ordenadores) con software apropiado.

El protocolo DICOM fue desarrollado originalmente por la "National Electrical Manufacturers Association (NEMA)" y el "American College of Radiology" para imágenes TC y RNM [19]. Actualmente está controlado por el "DICOM Standards Comité". El sistema DICOM usa TCP/IP (el estándar de Internet) como protocolo de transporte.

Se trata de un formato en expansión, que no se limita al campo del análisis de imagen. Así, por ejemplo, se han comunicado experiencias de integración de estos ficheros con programas de exposición de imágenes e información médica al objeto de poder emplearlos eficazmente en presentaciones médicas [20]. Hay "plug-in" publicados que pueden ser usados para cargar ficheros completos de pacientes en estas presentaciones o en aplicaciones concretas [2I].

La mayoría de los sistemas de imagen obtienen ficheros DICOM, pero existen equipos que generan formatos concretos. Para tales ficheros de imagen propietarios existen herramientas de conversión a DICOM [22].

Veamos ahora las principales técnicas de API que se realizan sobre estos ficheros.

Conviene distinguir, básicamente, entre dos tipos de sistemas aplicables en la virtopsia:

\section{Software de API:}

Son programas de ordenador que permiten efectuar los procedimientos de API.

Existen en el mercado múltiples aplicaciones comerciales que pueden realizar tareas de visualización DICOM y API $[23,24]$. Sin embargo, su coste no se encuentra al alcance de todos los centros, lo que no impide que podamos emplear otros procedimientos. 
Afortunadamente, abundan los programas que, además de leer ficheros DICOM haciendo posible su visualización, incorporan rutinas de API, permitiendo algunos de ellos generar o leer modelos tridimensionales. Unos son "freeware o shareware" [25,26,27,28,29], y otros comerciales. Algunos visores DICOM de dominio público, tienen, incluso, capacidad cliente-servidor [30].

Muchos equipos de CT y RNM disponen de software propio capaz de realizar estas tareas, incluso mediante reconstrucciones tridimiensionales. Sin embargo, nosotros pensamos que las tareas de API deben ser realizadas más en el lado del cliente (usuario) que del servidor (radiología) pues aquél es el que conoce para qué pretende emplearlas.

\section{Técnicas de proceso:}

Se trata de operaciones de índole eminentemente manipulativo, destinadas a modificar alguna de las características de la imagen con diferente finalidad, que puede ir desde la mejora de su calidad, hasta el realce de detalles poco visibles o la detección de elementos apenas visibles u ocultos al ojo humano. Estos procesos pueden incluir:

- Magnificación

- Filtrado

- Detección de bordes

- Manipulación de paletas

- Sustracción de fondos y de imágenes

- Reconstrucción (2-d, 3-d)

- Interacción de píxeles (división, multiplicación, suma, resta, etc.)

- Manipulación matemática de píxeles (mediante operaciones aritméticas o funciones)

- Segmentación

- Otros.

\section{Técnicas de análisis:}

Se trata de operaciones de índole mensurativo efectuadas sobre una imagen, y de cuyos resultados pretendemos obtener información acerca de algunas de las características del objeto u objetos a que corresponde dicha imagen.

Aquí priman los cálculos como elemento principal de lo analizado. No utilizamos funciones para manipular la imagen (esto sería más bien un proceso) sino que pretendemos obtener datos numéricos del objeto al que pertenece la imagen a través del análisis de ésta.

Podemos, así, medir su tamaño, su forma, su perímetro, el número de objetos determinados, su densidad óptica, su color, la distancia entre objetos o formas, ángulos, trayectorias, etc. Las técnicas podrán, así, incluir la:

- Morfometría

- Densitometría

- Reconstrucciones tridimensionales

- Otros procesos (contaje, colorimetría, etc.)

En la virtopsia pueden emplearse cualquiera de estas técnicas, tanto de análisis como de proceso. La ventaja es que podemos hacerlo interactivamente y sin alterar el modelo, que quedará siempre a nuestra disposición para continuar el análisis interactivo. 
Algunos han llamado visualización interactiva de imagen a la posibilidad de examinar los ficheros obtenidos por las técnicas de radiología digital, mediante una interactuación en tiempo real con las imágenes obtenidas. Esto es, en realidad, una combinación de las técnicas de proceso y de análisis de imagen pues podemos girar esta, realzarla, magnificarla, medirla, convertirla en un modelo tridimensional, que, a su vez, puede ser manipulado, etc. (Fotos I y 2).
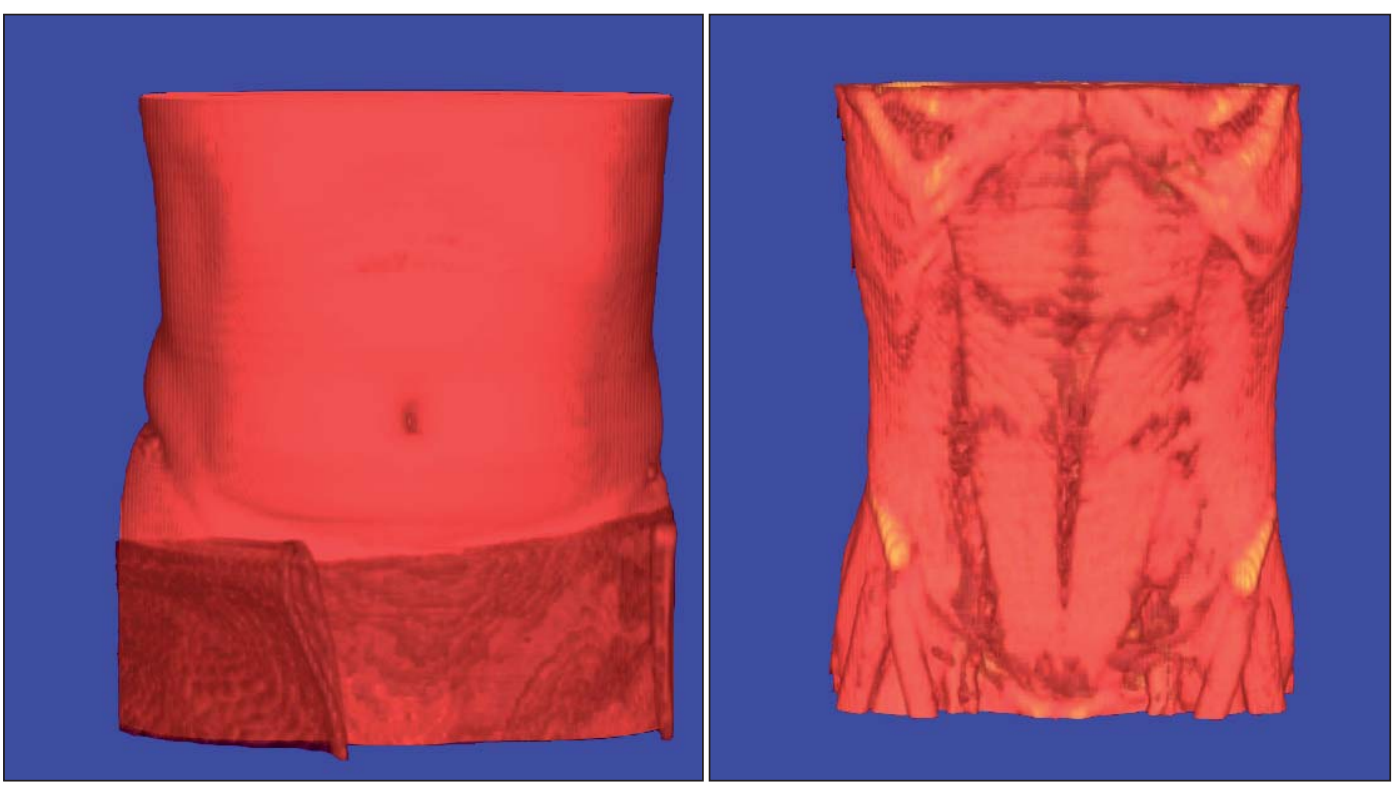

Figuras 1 y 2: Aspecto general del abdomen tras reconstrucción tridimensional de una TC abdominal. Nótese la perfecta definición de la piel y las referencias anatómicas. Es visible, incluso, la sabana cubriendo el pubis, lo que da idea de la definición de los datos contenidos en el DICOM. La imagen 2 muestra el aspecto de los músculos de la pared anterior abdominal tras segmentación y retirada virtual de la piel y el tejido celular subcutáneo.

Lo que permite dicha visualización interactiva es aplicar toda la potencia de los recursos de API al estudio de un fichero que contiene información del cuerpo, y efectuarlo en tiempo real.

Las aplicaciones de esta visualización en el campo forense, tanto en el vivo como en el cadáver, son inusitadas. Por ello, de la misma manera que un patólogo forense precisa conocer las técnicas macro y microscópicas de examen, la virtopsia requiere del prosector virtual que posea un profundo conocimiento de los recursos informáticos que posibilitan este tratamiento de la imagen digital.

Veamos ahora algunas de ellas.

\section{Técnicas 3D:}

Se han aplicado a múltiples campos en Medicina y Biología [3 I]. Entre ellas distinguimos principalmente dos tipos:

\section{RECONSTRUCCIONES:}

Las reconstrucciones tridimensionales son unas de las técnicas que producen resultados más espectaculares dentro del análisis de imagen vinculado a la autopsia (Fotos 3 y 4). Su empleo como elemento capaz de proporcionar información sobre las lesiones y también como patrón reconstructivo del suceso ha sido recientemente enfatizado [32]. 

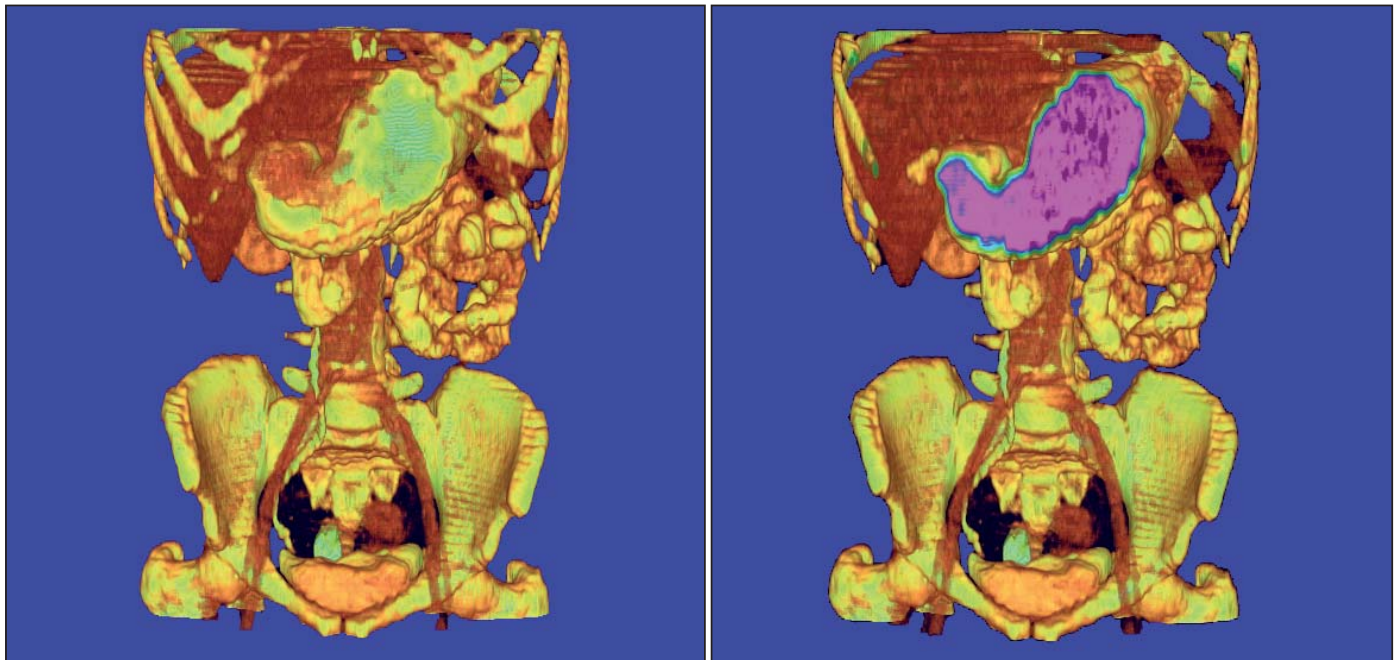

Figuras 3 y 4: Imágenes de virtopsia toraco-abdominal. En 3 son visibles la mayoría de las vísceras abdominales, así como estructuras retroperitoneales, como el uréter derecho y el intestino delgado. La exploración se realizó en sujeto vivo, con contraste baritado, de ahí que el estómago e intestino delgado aparezcan tan visibles. Igualmente es patente la aorta y la bifurcación ilíaca. En 4 se aprecia la mucosa gástrica al haberse cortado virtualmente el estómago.

Mediante este procedimiento podemos generar un modelo tridimensional del caso que, a su vez, puede ser sometido a visualización interactiva. Podemos procesarlo virtualmente, mediante cortes en el sentido que queramos, sustracciones de elementos como partes blandas, hueso, etc. (Fotos 5 y 6); pueden calcularse volúmenes, trayectorias, ángulos o cualquier otro elemento morfométrico, con el evidente interés que ello depara en la reconstrucción de los hechos y en la presentación en el foro. Estas técnicas pueden ser aplicadas además tanto en el cadáver como en el vivo, con lo que exceden el campo de actuación de la patología post-mortal para entrar de lleno en la valoración de daño corporal. Nosotros la hemos empleado en el análisis de fracturas espinales al objeto de averiguar su mecanismo de producción, con muy buenos resultados [33] (Foto 7).

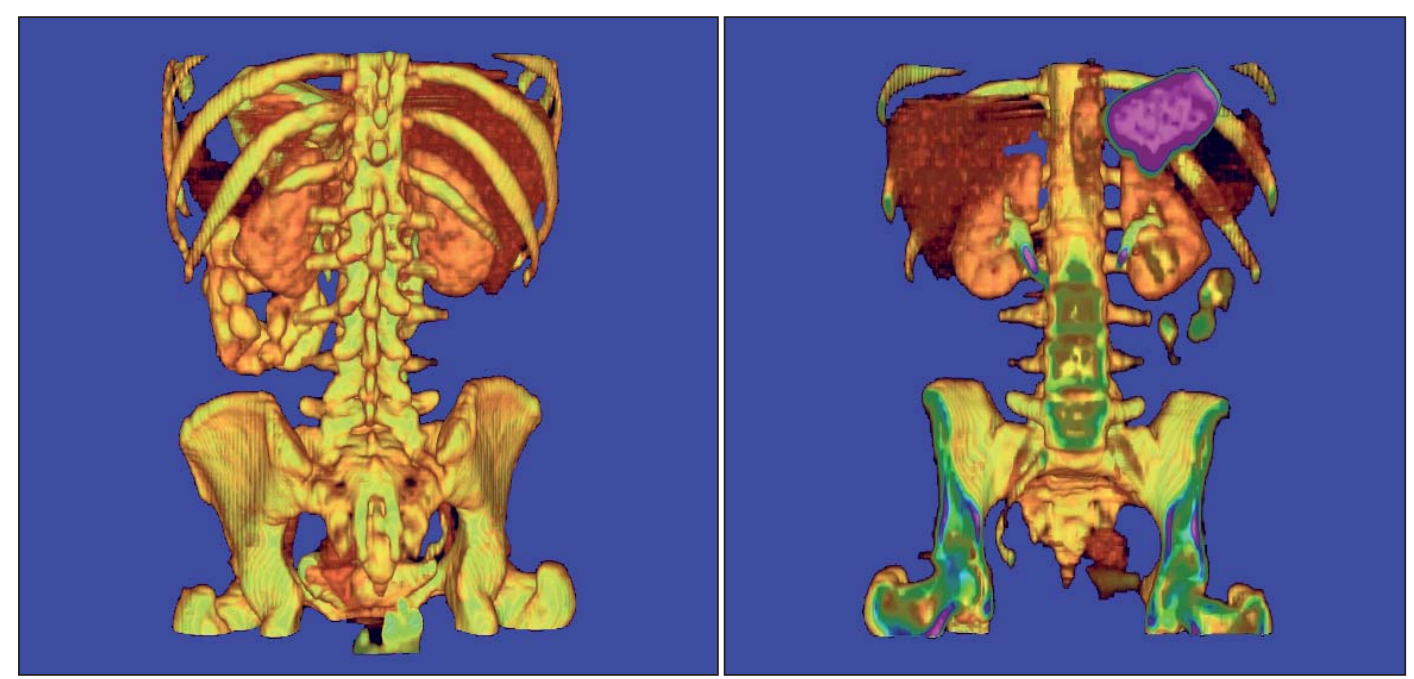

Figuras 5 y 6: Una de las ventajas de la virtopsia es la interactuación en tiempo real con el modelo, pudiendo someter a este a extracciones de elementos o a cortes o segmentaciones. En las imágenes se aprecia el esqueleto toraco-pelviano y diferentes órganos como el riñón desde ángulos distintos y perspectivas diferentes. 

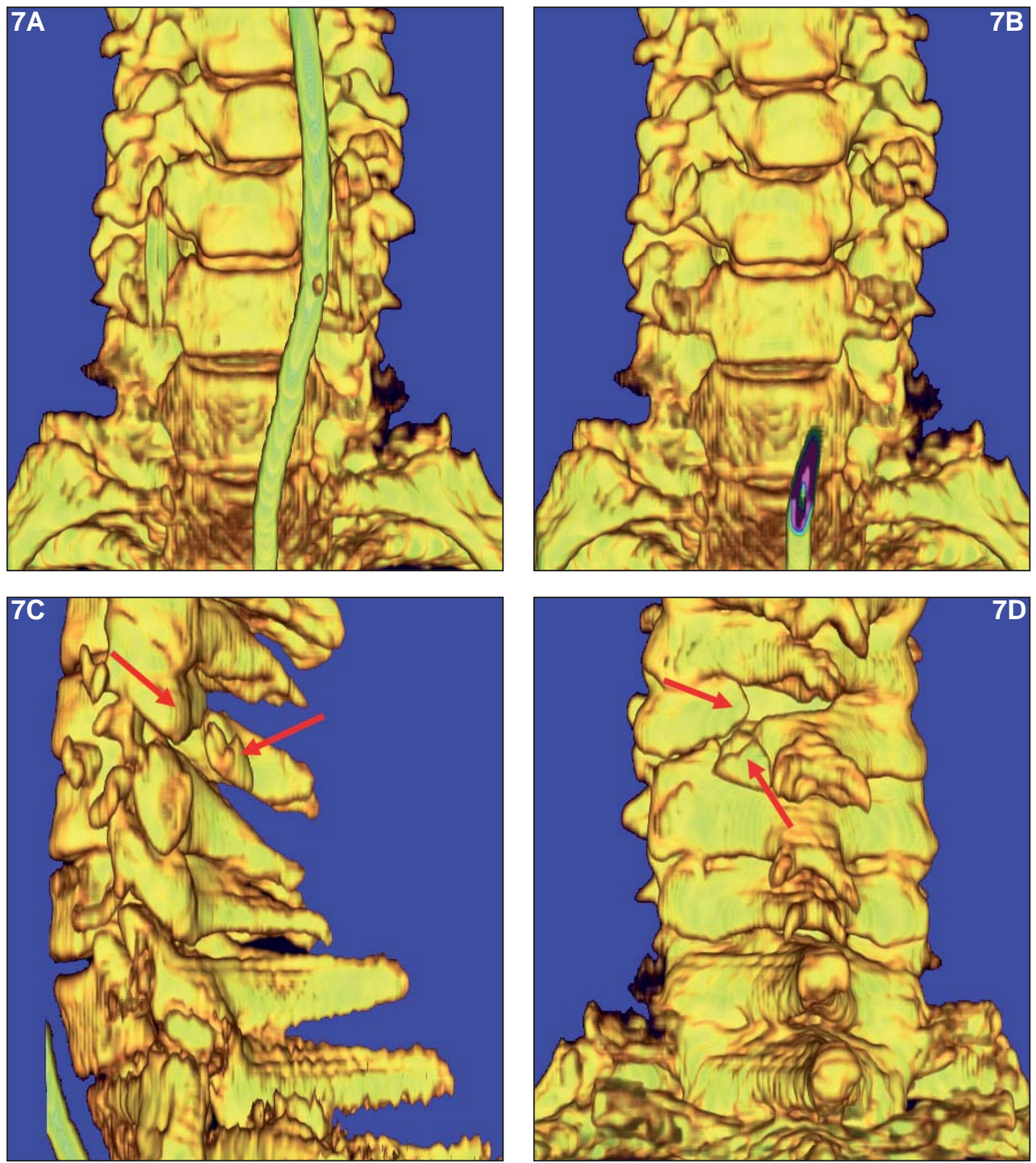

Figura 7a : Sujeto con tetraplejia de entrada tras accidente de circulación. Esta imagen muestra una reconstrucción tridimensional a partir de la TC. Se aprecia una anomalía de alineación C4-C5, pero no son visibles fracturas. La estructura circular en primer plano es la sonda naso-gástrica.

Figura 7b : Se ha efectuado un corte virtual de la sonda para poder ver las estructuras óseas. Es patente la luxación lateral de C4.

Figura 7c : Visión lateral del modelo tridimensional, en el que es visible una fractura articular en C4, con retropulsión de un fragmento (flechas).

Figura 7d : Visión posterior del mismo caso. Se aprecia claramente la fractura articular y de lámina de C4 (flechas).

También los ficheros de imagen contienen información acerca de la estructura molecular y mineral del espécimen con lo que podrían en un futuro a través de la reconstrucción adquirirse incluso las propiedades físico-químicas del objeto (resistencia, elasticidad, coeficiente de rozamiento, peso, deformabilidad, etc.). Ello es de gran importancia en reconstrucción 3D pues si sabemos, por ejemplo cuales son las características físicas de una cabeza conoceremos, por ejemplo que fuerza hay que aplicar para romper el hueso en ese caso, lo que seria enormemente útil desde el punto de vista medico-legal. 
También es posible, conocidas tales características, reconstruir en animación, por ejemplo un puñetazo que dio con el sujeto en el suelo o cualquier otro tipo de suceso con una gran fidelidad y resolver problemas tales como el modo de producción de unas lesiones craneales, etc.

En la actualidad estamos trabajando en un proyecto junto con el Centro Zaragoza de reconstrucción accidental aplicado a las lesiones del raquis cervical en accidentes de circulación.

\section{DRAMATIZACIONES}

Son recreaciones animadas más o menos fidedignas de un suceso a partir de los elementos de índole científica que puedan obtenerse del hecho. Es deseable que los datos se aproximen con la mayor exactitud a elementos cuantificables obtenidos del suceso. Es decir, que tantos más elementos de dicha índole tengamos menos podremos hablar de dramatización y más de reconstrucción. Pese a ello la dramatización resulta extraordinariamente útil en el contraste de varias hipótesis en un caso. En ocasiones esta simulación puede bastar para rechazar alguna de ellas, sin que se confirme, lógicamente ninguna, lo que siempre es mejor que nada. En ocasiones los testigos a los que se les presentan pueden proporcionar sobre la pantalla datos fiables en cuanto a cual de ellas es la más cercana a la realidad.

\section{Morfometría:}

Se trata de efectuar mediciones sobre una imagen relativas a parámetros relacionados con su forma y dimensiones, de los que podamos extraer aquellos análogos que corresponden al objeto al que pertenece la imagen. Ya que los ficheros DICOM contienen información muy precisa sobre calibración, podemos realizar sobre ellos todo tipo de mediciones (ángulos, distancias, perímetros, trayectorias, etc.). Estas mediciones resultan de enorme utilidad en el estudio forense de las lesiones.

La morfometría puede realizarse en el volumen tridimensional del cuerpo, con sus medidas reales, lo que supone una gran ventaja. Igualmente, puede interactuarse con el modelo para simular aperturas, segmentar regiones de interés, aislar órganos y un largo etcétera. La autopsia se convierte en algo virtual que no invalida, además, cualquier operación anatómica ulterior, sino que la dirige al poder anticipar sus hallazgos.

\section{Densitometría:}

Se basa en la existencia de una correspondencia entre la densidad óptica de la imagen (DO) y la intensidad o luminosidad de un pixel en la imagen.

En Antropología Forense el estudio de las densidades óseas puede ser relevante en el diagnostico de la data de la muerte [34].

\section{APLICACIONES DE LA VIRTOPSIA:}

\section{PATOLOGÍA FORENSE}

La principal aplicación en este campo es el estudio de las lesiones y de las causas de muerte.

La identificación de hematomas ocultos [7], el estudio del impacto, de trayectorias de lesiones (armas de fuego o armas blancas) pueden ser de suma utilidad en el esclarecimiento de un caso. Además, las mediciones morfométricas de las lesiones son exactas y permiten una exhaustiva documentación del caso, que puede ser sometido a reexamen tantas veces como queramos. 
La etiología y la causalidad lesional pueden ser estudiadas de una manera muy precisa. A título de ejemplo, una fractura craneal o espinal puede ser valorada de manera muy precisa en sus detalles morfopatológicos y puede generarse un modelo tridimensional real con lo que puede averiguarse el mecanismo de producción (Foto 8). También en lesiones de huesos largos resulta de extrema utilidad este modelado tridimensional.

\section{TANATOLOGÍA}

Se han publicado trabajos enfatizando la utilidad de la espectroscopia RNM en el estudio bioquímico del cadáver. Elementos como la data de la muerte pueden ser calculados en un futuro quizás a expensas de estos elementos bioquímicos que posibilitan una verdadera histoquímica no invasiva.

Ya señalamos antes la utilidad potencial de los procedimientos densitométricos en la averiguación de la data de restos óseos [34].

\section{IDENTIFICACIÓN}

\section{Superposiciones:}

Las superoposiciones fueron usadas por primera vez en 1935 para identificar restos esqueléticos [35]. Inicialmente la técnica incluía la superposición de una transparencia del cráneo sobre una fotografía ante-mortem del sujeto que ha sido reescalada al tamaño correcto y tomada desde los mismos ángulos fotográficos que aquélla [36]. Los dos principales pro-

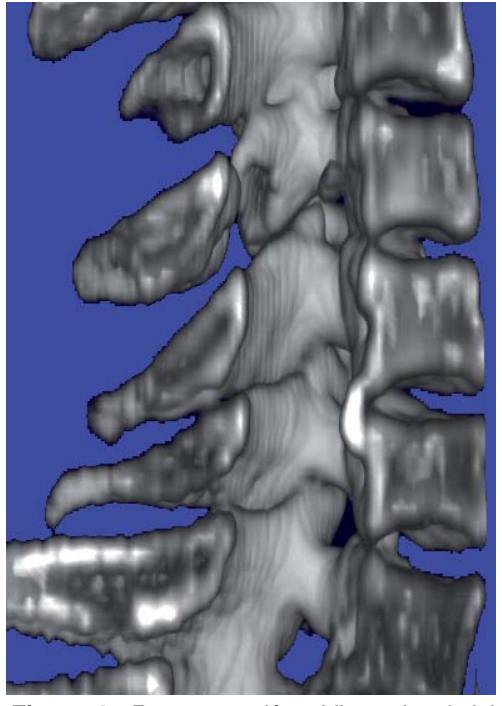

Figura 8: Reconstrucción tridimensional del caso de la foto 7. Se ha efectuado un corte virtual sagital que nos permite identificar un fragmento óseo dentro del canal medular en $\mathrm{C4}$, verosimilmente procedente del estallido articular, así como un osteofito C5-C6. Esta virtopsia del caso permite hacerse una idea exacta de las lesiones y de las causas de una tetraplejia que, a juzgar por las lesiones óseas, es difícilmente explicable. Probablemente, un mecanismo de flexión-distracción produjo un daño medular completo, por mecanismo de cizallamiento, retornando después la hemivértebra luxada a su posición inicial, dejando como testigo de su traslación el pequeño fragmento mencionado. blemas de estas técnicas cuando partimos de fotografías y radiografías son:

I.- El factor de magnificación. Las dos imágenes (ante y postmortem) no han sido realizadas a igual distancia ni con análogo zoom. Es necesario, por tanto, reescalar una fotografía al tamaño de la otra.

2.- El paralaje. La imagen del cráneo debe ser tomada en el mismo ángulo que lo fue la fotografía original.

Para solventar el primero de estos problemas se han usado numerosos procedimientos. Se han empleado los objetos que se sitúan en el mismo plano de la fotografía (objetos personales como la corbata, las gafas, etc...) pueden utilizarse para reescalar la imagen del cráneo hasta las dimensiones de la fotografía ante-mortem. Esto tiene el inconveniente de que si el objeto utilizado no se halla en el mismo plano que la cara no podría se empleado para el reescalado pues la distancia no sería la misma.

El empleo de imagen digital procedente de técnicas radiológicas permite la corrección del paralaje pues podemos girar el modelo tridimensional o multiplanar en el sentido apropiado y superponerlo con la imagen problema. También es posible la realización de morfometrias comparativas entre los datos de la técnica de imagen y la iconografía previa del sujeto. 
La TC y la RNM permiten visualizar el rostro del sujeto en un modelo tridimensional, lo que podría aplicarse en el futuro a la realización de esculturas forenses virtuales a efectos identificativos.

\section{ANTROPOLOGÍA FORENSE}

La morfometría y densitometría tienen evidente interés aquí. Los modelos procedentes de los DICOM obtenidos por TC pueden ser sometidos a mediciones de todos los parámetros antropológicos (Foto 9). La densitometría que proporciona la TC informa en detalle de la mineralización del hueso, por lo que puede aplicarse a cálculos de data de la muerte.
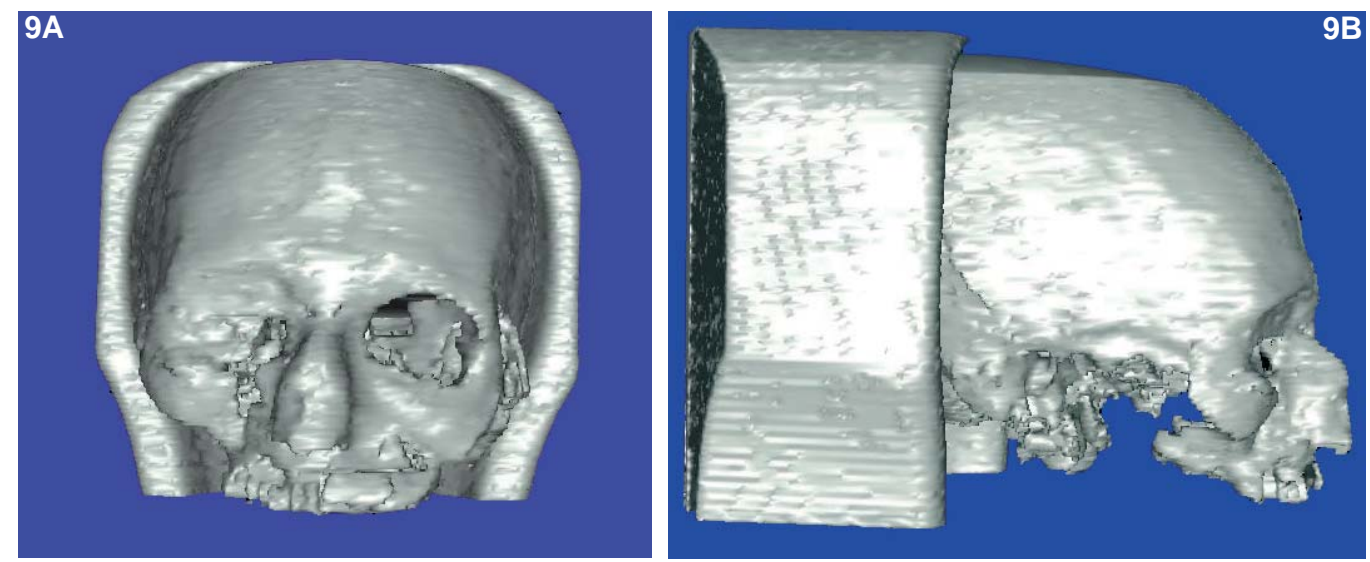

Figura 9a y 9b: Normas frontal y lateral de la virtopsia del cráneo de Benedicto XIII. La reconstrucción se efectuó a partir de un estudio TC. Sobre este modelo se efectuaron todo tipo de mediciones antropométricas y otras tareas de API cara a su identificación. El modelo virtual fue cortado, de manera que pudieran efectuarse inspecciones de su interior sin necesidad de abrirlo.

\section{VALORACIÓN DE DAÑO CORPORAL}

En valoración de daño, las principales aplicaciones son las siguientes:

- Morfometría de lesiones (trayectorias, dimensiones, ángulos áreas, volúmenes, etc.)

- Estudio de causalidad médico-legal

- Estudio de los mecanismos de producción de las fracturas

\section{VENTAJAS:}

Las principales ventajas del protocolo DICOM pueden resumirse en:

I.- Estandarización y universalización de los ficheros gráficos del diagnóstico por imagen en Medicina.

2.- Posibilidad de interrogación de servidores DICOM desde terceras partes (búsqueda de imágenes de manera interactiva y organizada).

3.- Posibilidad de acceso remoto a bases DICOM (tele-diagnóstico y teletratamiento).

4.- Acceso a la información no gráfica de los ficheros (calibración en las dimensiones reales del paciente, densidades, número de imágenes, resolución de estas, dimensiones de los cortes, etc.).

5.- Posibilidad de exploración y visualización interactivas de los ficheros: algo así como un "negatoscopio virtual".

6.- Precisión en las técnicas de morfometría lesional.

7.- Posibilidad de repetición de las técnicas mediante interacción con un modelo las veces que sea preciso. 


\section{INCONVENIENTES:}

I.- Necesidad de infraestructura de radiología digital

2.- Necesidad de desplazar cadáveres o piezas al Servicio de radiología digital

3.- Entrenamiento en el empleo de las herramientas de software apropiadas

Como puede verse, la sofisticación de estas técnicas reduce significativamente sus posibilidades de empleo en la rutina forense de nuestro país. No obstante, conviene señalar que muchos de los casos autopsiados ha sido sometidos en los hospitales previamente a su fallecimiento a este tipo de técnicas. Así, es posible contar con tales estudios en formato DICOM, podrían ser examinados con técnicas de virtopsia bien como complemento de la autopsia tradicional o como contraprueba, lo que resulta necesario conocer.

\section{CONCLUSIONES:}

I.- La virtopsia es un procedimiento de examen forense, basado en las técnicas de radiología digital y análisis de imagen.

2.- Puede aplicarse al sujeto vivo y al cadáver, en el ámbito de la Patología y Traumatología Forenses, Antropología y Valoración de daño corporal.

3.- Si bien son técnicas sofisticadas que requieren de infraestructura tecnológica y entrenamiento en recursos digitales, es preciso que el Médico Forense conozca la existencia y sus aplicaciones dentro del campo de la Medicina Legal.

4.- Los procedimientos de virtopsia se encuentran un horizonte tanto menos lejano cuanto más impulsemos su introducción en el ámbito de la medicina forense.

\section{BIBLIOGRAFÍA:}

I. Garfia A. Técnicas de autopsia en el fin del milenio. Las opsias. Panorama general e indicaciones. Curso sobre "Técnica avanzada de autopsia". Centro de Estudios Judiciales. Administración de Justicia. Madrid 26-28 de octubre de 1998.

2. Thali MJ, Yen K, Schweitzer W, Vock P, Boesch C, Ozdoba C, Schroth G, Ith M, Sonnenschein M, Doernhoefer T, Scheurer E, Plattner T, Dirnhofer R., Virtopsy, a new imaging horizon in forensic pathology: virtual autopsy by postmortem multislice computed tomography (MSCT) and magnetic resonance imaging (MRI)--a feasibility study. J Forensic Sci 2003 Mar;48(2):386-403.

3. Plattner T, Thali MJ, Yen K, Sonnenschein M, Stoupis C, Vock P, Zwygart-Brugger K, Kilchor T, Dirnhofer R. Virtopsy-postmortem multislice computed tomography (MSCT) and magnetic resonance imaging (MRI) in a fatal scuba diving incident. J Forensic Sci 2003 Nov;48(6):1347-55.

4. Aso J, Arregui R, Calatayud JB, et al. Análisis de imagen y planificación operatoria. Su integración en la rutina neuroquirúrgica. Comunicación presentada al Congreso de la Sociedad Española de Neurocirugía. Santiago de Compostela. 2002.

5. Oliver WR. Image processing in Forensic Pathology. Clinics In Laboratory Medicine 1998, I8(I):15I-180.

6. Aghayev E, Thali M, Jackowski C, Sonnenschein M, Yen K, Vock P, Dirnhofer R. Virtopsy-fatal motor vehicle accident with head injury. J Forensic Sci 2004 Jul;49(4):809-I3.

7. Yen K, Vock P, Tiefenthaler B, Ranner G, Scheurer E, Thali MJ, Zwygart K, Sonnenschein M, Wiltgen M, Dirnhofer R. Virtopsy: forensic traumatology of the subcutaneous fatty tissue; multislice computed tomography (MSCT) and magnetic resonance imaging (MRI) as diagnostic tools. J Forensic Sci 2004 Jul;49(4):799-806.

8. Jackowski C, Schweitzer W, Thali M, Yen K, Aghayev E, Sonnenschein M, Vock P, Dirnhofer R. Virtopsy: postmortem imaging of the human heart in situ using MSCT and MRI. Forensic Sci Int 2005 Apr 20;149(I):II-23.

9. Jackowski C, Thali M, Sonnenschein M, Aghayev E, Yen K, Dirnhofer R. Adipocere in postmortem imaging using multislice computed tomography (MSCT) and magnetic resonance imaging (MRI). Am J Forensic Med Pathol 2005 Dec;26(4):360-4.

10. Jackowski C, Sonnenschein M, Thali MJ, Aghayev E, von Allmen G, Yen K, Dirnhofer R, Vock P. Virtopsy: postmortem minimally invasive angiography using cross section techniques--implementation and preliminary results. J Forensic Sci 2005 Sep;50(5):II75-86.

II. Jackowski C, Thali M, Aghayev E, Yen K, Sonnenschein M, Zwygart K, Dirnhofer R, Vock P. Postmortem imaging of blood and its characteristics using MSCT and MRI. Int J Legal Med 2005 Nov; $19: 1-8$.

I2. Johnson, [p, Lawler W Burns ]. Use of hystomorphometry in the assessment of fatal vertebral artery dissection. J Clin Pathol 1993; 46(II): 1000-1003. 
13. Giebe W, Ahne R, Huhn P, Laumer N. Chromosomenanalyse zur vaterschaftsfeststellung mit dem bildauswertesystem AMBA/RChro-H. Beitr Gerichtl Med 1989; 47: 563-569.

14. Facey Oe, Hannah Id, Rosen D. Shoe wear patterns and pressure distribution under feet and shoes, determined by image analysis. J Forensic Sci Soc 1992; 32(I): 15-25.

15. Lopez-Nicolas M, Luna A. Application of automatic image analysis (IBAS system) to age calculation. Efficiency in the analysis of several teeth from a single subject Forensic Sci Int 199I; 50(2): 195-202.

16. Xu, Xh, Philipsen Hp, Jablonski Ng, Weatherhead B, Pang Km, Zhu Jh. Preliminary report on a new method of human age estimation from single adult teeth Forensic Sci Int 1994;5I(2): 28I288.

17. Kintzl HP, Thieme J, Schreiber H, Geissler D, Grim 0. Computergestutze identifizierung und vergleichsuntersuchungen menslicher schadel, Beitr Geritch Med 1990; 48: 27-29.

18. Rorden C. The DICOM standard. Disponible en: http://www.psychology.nottingham.ac.uk/staff/crl/dicom.html. Accedido el 24-10-2005.

19. NEMA. Digital imaging and communications in medicine (DICOM). Tech Rep. National Electrical Manufacturers Association, 200I. Disponible en:

http://medical.nema.org/dicom/2000.html. Accedido el 4-II-2005. 20. Haider MA. Extending PowerPoint with DICOM image support. Radiographics 2003;23(6):1683-1687.

2I. Rorden C. Active $X$ and CLX DICOM components. Disponible en: http://www.sph.sc.edu/comd/rorden/dicomcom.html\#Source. Accedido el 24-10-2005.

22. David Clunie's Medical Image Format Site. Dicom3tools Software. Disponible en:

http://www.dclunie.com/dicom3tools.html. Accedido el 3-II-2005.

23. 3D Doctor. Disponible en:

http://www.ablesw.com/3d-doctor. Accedido el 23-10-05.

24. Mevislab. Medical Image Processing and Visualization. Disponible en: http://www.mevislab.de/. Accedido el 25-10-05.
25. Simple DICOM Receiver \& Viewer. Disponible en: http://www.radiology.pitt.edu/Public/public_resources/software/s impleDICOM/ReceiverViewer/index.html. Accedido el 3-II-2005.

26. Image J. Bio-medical Imaging in Java. Disponible en: http://bij.isi.uu.nl/index.htm. Accedido el 3-II-2005.

27. MRicro. Disponible en:

http://www.sph.sc.edu/comd/rorden/mricro.html. Accedido el 3II-2005.

28. Free medical imaging software. Disponible en: http://www.idoimaging.com/index.shtml. Accedido el 3-II-2005.

29. Medical Volume Explorer. Disponible en: http://www.medical-image-processing.info/MVE/. Accedido el 23$10-05$.

30. Conquest DICOM. Disponible en: http://www.xs4all.nl/ ingenium/dicom.html. Accedido el 3-II-2005.

3I. Robb, RA. Three-Dimensional Visualization in Medicine and Biology. En: Bankman IN. Handbook of Medical Imaging: Processing and Analysis. Academic Press, 2000 pp. 685-712.

32. Thali MJ, Braun M, Buck U, Aghayev E, Jackowski C, Vock P, Sonnenschein M, Dirnhofer R. VIRTOPSY--scientific documentation, reconstruction and animation in forensic: individual and real 3D data based geo-metric approach including optical body/object surface and radiological CT/MRI scanning. J Forensic Sci 2005 Mar;50(2):428-42.

33. Aso J, Martínez Quiñones, JV, Arregui, R, De Miguel, JL, Olona, A, Pons, J. Reconstrucción 3D sobre ficheros DICOM. Su utilidad en el estudio causal en Valoración de Daño corporal. Segundo Premio a la mejor comunicación en las VI Jornadas Nacionales de Valoración de Daño corporal. Fundación Mapfre Medicina. Madrid. 2005.

34. Baena S. Densitometría y data de la muerte. Tesis Doctoral. Universidad de Zaragoza. Junio de 2001.

35. Glaister J, Brash JC. Medico-legal aspects of the Ruxton case. E\&Livingstone. Edimburgh, 1937 pp. 140-170.

36. Chee LF, Cheng CT. Skull and photographic superimposition. A New approach using a second-party's interpupil distance to extrapolate the magnification factor. J Forensic Sci 1989; 34, 3:708-713. 